\title{
Cardiopulmonary and hemodynamic changes in complement activation-related pseudoallergy
}

\author{
László Dézsii ${ }^{{ }^{*}}$, Gábor Szénási ${ }^{2}$, Rudolf Urbanics ${ }^{3}$, László Rosivall ${ }^{2,4}$, János Szebeni ${ }^{1,5}$ \\ ${ }^{1}$ Nanomedicine Research and Education Center, Semmelweis University, Budapest, Hungary; \\ *Corresponding Author: dezsi.laszlo@med.semmelweis-univ.hu \\ ${ }^{2}$ Institute of Pathophysiology, Faculty of Medicine, Semmelweis University, Budapest, Hungary \\ ${ }^{3}$ SeroScience Ltd., Budapest, Hungary \\ ${ }^{4}$ Hungarian Academy of Sciences-Semmelweis University, Nephrology Research Group, Budapest, Hungary \\ ${ }^{5}$ Department of Pathophysiology, Faculty of Health, Miskolc University, Miskolc, Hungary
}

Received 7 March 2013; revised 8 April 2013; accepted 9 May 2013

Copyright (C 2013 László Dézsi et al. This is an open access article distributed under the Creative Commons Attribution License, which permits unrestricted use, distribution, and reproduction in any medium, provided the original work is properly cited.

\begin{abstract}
Complement activation-related pseudoallergy (CARPA) is a frequent side effect of intravenous therapies with nanoparticle-containing drugs and biologicals that are recognized by the immune system as foreign. It is an acute infusion reaction dominated by cutaneous and hemodynamic changes, most significantly a cardiopulmonary distress involving major pulmonary hypertension, systemic hypotension and arrhythmias. Because CARPA is unpredictable by conventional allergy tests and it may be life threatening, it can represent a major barrier to the safe therapeutic application of many modern medicines, including liposomal drugs and monoclonal antibodies. This review summarizes and updates the facts and opens questions regarding this phenomenon, with particular focus on its porcine model.
\end{abstract}

Keywords: CARPA; Hypersensitivity; Anaphylaxis; Liposome; Pig

\section{INTRODUCTION}

A causal relationship between immune changes and cardiovascular diseases is a well known phenomenon, for example, immune involvement in chronic heart failure via myocyte damage [1]. Another cause-effect relationship between the two systems is exemplified by drug and food allergies, or insect bites, which lead to the rapid development of a wide range of severe cardiopulmonary changes that can cause cardiac (anaphylactic) shock and death. Symptoms, e.g. arrhythmia, pulmonary edema, hy- potension, airway occlusion, respiratory distress and cardiac arrest are also typical of hypersensitivity reactions (HSRs) to certain drugs, where the underlying cause is the activation of the complement (C) system. These reactions were called $\mathrm{C}$ activation-related pseudoallergy (CARPA) [2].

\section{DIFFERENT FORMS OF HSRS: CLASSICAL ANAPHYLAXIS AND CARPA}

HSRs have been categorized by Coombs and Gell in four groups from I to IV [3]. Type I reactions are defined as IgE-mediated acute reactions, while the rest of categories included subacute or chronic immune changes triggered or mediated by IgG, immune complexes or lymphocytes. However, it has increasingly been recognized that a substantial portion of acute allergic reactions, whose symptoms fit in Coombs and Gell's Type I category, are actually not initiated or mediated by pre-existing IgE antibodies. To distinguish between classical anaphylactic reactions and these non-IgE-mediated ones, the latter category was termed pseudoallergy [4]. Pseudoallergy could be elicited by various agents e.g. radio-contrast media (RCM), nonsteroidal anti-inflammatory drugs, analgesics, morphine and insect venoms, liposomes and micellar solvents, such as Cremophor EL (CrEL) in Taxol [2]. There are numerous data suggesting that $C$ activation is a common trigger mechanism of these reactions, where C3a and C5a activation products are generally involved [4]. The term, CARPA, was suggested on the basis of this evidence [5].

Some symptoms of CARPA are the same as seen in common allergy or classical Type I reactions, while others are unique to $\mathrm{C}$ activation (Table 1). The most im- 
portant distinguishing feature of CARPA seems to be the lack of pre-sensitization and reinforcement, that is already the first exposure to a given noxious event or drug elicits maximal and in some cases fatal cardiovascular reaction. Upon repeated exposure the response decreases, frequently tachyphylaxis develops [6,7].

According to novel evidence summarized above a new scheme of hypersensitivity reactions was proposed by Szebeni et al. [2,5] with revision of the Type I category of Coombs and Gell's classification (Figure 1).

\section{RELEVANCE OF CARPA IN HUMANS}

The clinical picture of CARPA includes cardiovascular (tachycardia or bradycardia, arrhythmia, hypo- and hypertension, chest pain, back pain), respiratory (tachypnea, bronchospasm, dyspnea), and cutaneous (flushing, urticaria, erythema, pruritus) symptoms.

Table 1. Symptoms of IgE-mediated Type I allergy and C activation-related pseudoallergy (CARPA).

\section{Common symptoms}

Angioedema, asthma attack, bronchospasm, chest pain, chill, choking, confusion, conjunctivitis, coughing, cyanosis, death, dermatitis, diaphoresis, dispnoea, edema, erythema, feeling of imminent death, fever, flush, headache, hypertension, hypotension, hypoxemia, low back pain, lumbar pain, metabolic acidosis, nausea, pruritus, rash, rhinitis, shock, skin eruptions, sneezing, tachypnea, tingling sensations, urticaria, wheezing

\section{Unique symptoms}

IgE-mediated Type I

Reaction arises after repeated exposure to the allergen

Reaction is stronger upon repeated exposures

Reaction does not cease without treatment

Reaction rate is low $(<2 \%)$

CARPA

Reaction arises at first treatment (no prior exposure to allergen)

Reaction is milder or absent upon repeated exposures

Spontaneous resolution

High reaction rate (up to $45 \%$ ), average $7 \%$, severe $2 \%$

Modified after Szebeni et al. [2].

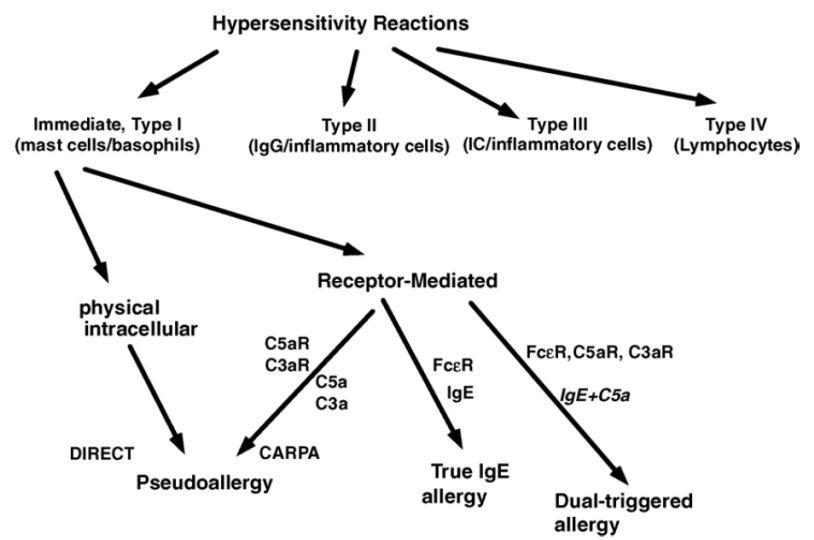

Figure 1. Proposed new scheme of hypersensitivity reactions (Szebeni et al. [2]).
The frequency of CARPA in the 5\% - 45\% range is much higher than classical anaphylactic reactions to drugs (for example, penicillin allergy occurs in $<2 \%$ ) [2]. Severe reactions can cause major anxieties, disruptions and exclusion of the patient from receiving a potentially useful life-saving treatment. These reactions can also be fatal in a small percentage of hypersensitive individuals, mainly those with a history of severe allergy and/or heart disease [8]. Complement activation during cardiopulmonary bypass with the involvement of C3a and C5a anaphylatoxins was reported decades ago [9], which was later confirmed and the involvement of C-reactive protein in association with postoperative arrhythmia was also described [10].

In the late 1990s PEG binding to the red blood cell (RBC) membrane was performed to cover red cell antigens, suggesting that such RBCs might be used as universal/stealth RBCs, suitable for transfusion. However, in vivo studies in rabbits and in vitro testing of human sera were discouraging because of antigenicity and/or immunogenicity of PEG-RBCs, negating the benefits of masking RBC blood group antigens with PEG. Later on similar problems have been found with PEGylated liposomes [11], including a study by Brouwers et al. (2000) [12], which reported 3 severe infusion reactions out of 9 patients obtaining Tc-99 m-PEG-liposomes for imaging of Crohn's disease. The study dedicated to correlate C activation with HSRs gave account of $45 \%$ reaction rate in cancer patients infused with the liposome encapsulating doxorubicine (Doxil) for the first time [13].

\section{HEMODYNAMIC EFFECTS OF COMPLEMENT ACTIVATION IN ANIMAL MODELS}

It has long been known that the lipopolysaccharideinduced immediate and reversible thrombocytopenia and hypotension were prevented by prior depletion of the C3 with the anticomplementary protein from cobra venom in conscious rabbits [14-16]. Later on it was also demonstrated that the anaphylatoxin C5a caused a reversible systemic arterial hypotension and decreased cardiac output while increased central venous pressure in control and neutropenic anesthetized rabbits. These hemodynamic effects were accompanied by increases in plasma prostanoid levels and neutropenia. Indomethacin abolished the C5a-induced hypotension. On the other hand, the thromboxane synthase inhibitor dazoxiben did not alter the hypotensive response but decreased changes in central venous pressure and decreased total peripheral circulatory resistance after $\mathrm{C} 5 \mathrm{a}$ administration. Cimetidine, a $\mathrm{H}_{2}$-receptor antagonist attenuated the C5a-induced hypotension and diminished prostanoid release [17].

A different way of activation of the alternative $C$ pathway by intravenous injection of zymosan caused a range 
of hemodynamic responses in anesthetized rats. Zymosan, at an intravenous dose of $2 \mathrm{mg} / \mathrm{kg}$, increased extravasation of ${ }^{125}$ I-labelled albumin in the lung. The zymosan-induced response was not restricted to the lung since the hematocrit was also increased. The peak effect of zymosan was observed $15 \mathrm{~min}$ after treatment at the highest dose of $16 \mathrm{mg} / \mathrm{kg}$ i.v., and extravasation of plasma was still significant $60 \mathrm{~min}$ after treatment. The vascular permeability change in the lung was abolished by pre-treatment with the cyclooxygenase inhibitors, indomethacin or ketoprofen and the lipoxygenase and cyclooxygenase inhibitor, BW 755C, and no plasma extravasation was found in rats made leucopenic by rabbit anti-neutrophil serum. On the other hand, the PAF antagonist, WEB 2086, the antihistamine, mepyramine or the non-selective serotonin antagonist, methysergide did not affect the response to zymosan [18].

The dose-dependent hemodynamic and respiratory effects of zymosan began with a transient increase in right ventricular systolic pressure and respiratory rate, and then a drop in blood pressure was noted lasting for 15 $30 \mathrm{~min}$ at the doses of 3 and $8 \mathrm{mg} / \mathrm{kg}$. The right ventricular systolic pressure decreased in parallel with the blood pressure at the higher dose of zymosan. WEB 2086 largely prevented the decreases in blood pressure and right ventricular systolic pressure, while indomethacin attenuated the tachypnea and the increase in right ventricular systolic pressure but augmented the drop in blood pressure and right ventricular systolic pressure. Complement activation was verified by reduced $\mathrm{C}$ hemolytic activity. These results demonstrated that in the rat, $C$ activation by zymosan lead to prostanoid-mediated pulmonary and PAF-mediated systemic vascular effects [18].

\section{HEMODYNAMIC EFFECTS OF LIPOSOMES AS A CONSEQUENCE OF COMPLEMENT ACTIVATION}

One of the first observations with nanoparticles was made with liposome-encapsulated hemoglobin (LEH) using hydrogenated soy lecithin. Injection of LEH in the conscious rat elevated mean arterial pressure and heart rate but decreased platelet count with a proportional increase in plasma $\mathrm{TXB}_{2}$ concentration. Liposome vehicles decreased blood pressure and platelet count but increased heart rate and plasma $\mathrm{TXB}_{2}$ levels. All effects were transient [19]. Using synthetic distearoyl phosphatidylcholine-based liposomes for encapsulating hemoglobin markedly diminished the hemodynamic effects. Injection of the improved preparation caused only a small tachycardia and elevation in plasma $\mathrm{TXB}_{2}$ level [20]. Pretreatment with the PAF antagonist BN 50739 abolished all changes caused by the distearoyl phosphatidylcholine- based liposome-encapsulated hemoglobin [21].

A big advance in understanding the drug-induced hypersensitive reactions was made by the discovery that intravenous injection of hemoglobin-containing liposomes and to a lesser extent empty liposomes but not hemoglobin decreased the plasma $\mathrm{C}$ hemolytic activity and increased the plasma $\mathrm{TXB}_{2}$ level. EGTA/ $\mathrm{Mg}^{2+}$ did not inhibit $\mathrm{C}$ consumption induced by hemoglobin-containing liposomes in rat plasma but pre-heating rat plasma to $50^{\circ} \mathrm{C}$ abolished $\mathrm{C}$ consumption. Treatment with the soluble $\mathrm{C}$ receptor type 1 (sCR1) or $\mathrm{C}$ depletion with the cobra venom factor decreased the liposome-induced rise in plasma $\mathrm{TXB}_{2}$ level. These results suggested that hemoglobin containing liposomes activated the alternative $\mathrm{C}$ pathway [22,23].

Since liposomes do not contain proteins, they likely activate the innate immunity. In fact, the size and shape of liposomes are similar to those of pathogenic viruses, nanobacteria and subcellular organelles. Therefore, liposomes are recognized by the nonspecific immune system as pathogens, so liposomes and other nanoparticles can activate efficient eliminatory mechanisms [24].

The pioneering findings related to CARPA were followed by the demonstration that liposomal drugs or liposomes, in general, caused immediate cardiovascular hypersensitivity reactions in anesthetized pigs. Intravenous injection of large multilamellar liposomes raised pulmonary arterial pressure, heart rate, pulmonary vascular resistance, systemic vascular resistance while caused a decrease in cardiac output $[4,25]$. These effects were dose-dependent and repeatable for several times in the same animal, exhibiting no tachyphylaxis. The rises in pulmonary arterial pressure correlated with elevations in plasma $\mathrm{TXB}_{2}$ levels. The cardiovascular hypersensitivity reactions were inhibited by an anti-C5a monoclonal antibody, sCR1, or indomethacin. The liposome-induced increase in C5a production was mediated via the classical C activation pathway and liposomes bound IgG and IgM natural antibodies in pig serum in vitro. Zymosan and hemoglobin-containing liposomes and empty liposomes caused nearly equal pulmonary artery pressure changes $[4,25]$.

Further studies identified that multilamellar liposomes, doxorubicin-containing liposomes (Doxil) or amphotericine B-containing liposomes (Ambisome) evoked cardiac anaphylaxis in pigs. Intravenous injection of liposomes rapidly resulted in dose-dependent hemodynamic (systemic hypotension, pulmonary hypertension, decreased cardiac output), respiratory (decreased endtidal $\mathrm{pCO}_{2}$ ) and mild to severe cardiac changes such as tachycardia and/or paradoxical bradycardia, arrhythmias, ST depression, T-wave elevation, atrioventricular block, ventricular fibrillation, and cardiac arrest (Figure 2). The severity of the cardiac effects significantly correlated 
with the liposome-mediated production of C5a in pig serum in vitro. Zymosan and recombinant human (rhu) C5a almost fully reproduced the above effects while pre-treatment with sCR1, an inhibitor of C activation via the classical and alternative pathways, or with the antiporcine C5a antibody GS1 markedly reduced the pulmonary and cardiac abnormalities [26].

Similar responses were obtained in anaesthetized rats. Intravenous injection of high cholesterol (71 mole \%) containing multilamellar liposomes at low dose (8 mg/kg) induced a rapidly developing drop in blood pressure, bradycardia, arrhythmia and tachypnea with clear signs of pulmonary edema. These responses faded in 10 - 30 min and repeated injections elicited a similar response. Blockade of the $\mathrm{C}$ system with cobra venom prevented the hypotensive response even after the injection of a lethal dose of liposomes (30 mg/kg). Pre-treatment with SQ 30,741, a TXA 2 receptor inhibitor was also protective against the liposome-induced cardiopulmonary distress [28].

\section{SPECIES DEPENDENCE OF THE LIPOSOME-INDUCED CARPA}

Comparison of the liposome-induced or nanoparticle-dependent CARPA across species revealed strong interspecies variability [8]. Liposomes with high cholesterol-content (71 mole \%) consumed $18 \%$ of total hemolytic $\mathrm{C}$ activity in rat sera, while the same liposome preparation consumed $100 \%$ of the activity in pig sera and more than $70 \%$ of the activity in human sera, in vitro. Cholesterol-poor liposomes consumed approximately only $2 \%$ of total hemolytic C activity in rat sera, but as much as $20 \%$ of pig and human hemolytic activity [28].

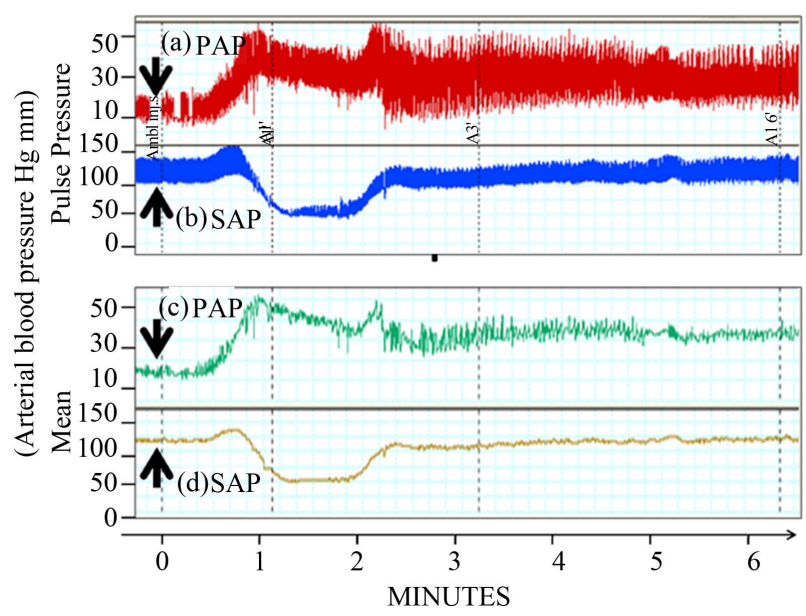

Figure 2. Cardiopulmonary changes following intravenous administration of Ambisome at $0.01 \mathrm{mg} / \mathrm{kg}, 80 \mathrm{nM}$ phospholipid/kg. PAP, pulmonary arterial pressure; SAP, systemic arterial pressure; arrows indicate the time of injection (Szebeni, et al. [27]).
The most sensitive cardiopulmonary distress model for the detection of CARPA in vivo is the anesthetized pig established in our laboratory (see experimental arrangement in Figure 3). Testing liposomes and nanoparticles in pigs can best predict human $\mathrm{C}$ activation and reactivity.

\section{DEPENDENCE OF CARPA-INDUCING ACTIVITY ON THE CHARACTERISTICS OF LIPOSOMES}

The liposome-induced C activation is highly dependent on the liposome characteristics. The reactivity of various liposome preparations can be evaluated by measuring the formation of S protein-bound C-terminal complex (SC5b-9) in normal human serum, in vitro [27]. It has been clearly shown that drug-loaded liposomes caused much stronger reactions than empty ones. The free drug binds to the liposome surface, which alters the surface characteristics of the liposome leading to enhanced $\mathrm{C}$ activation. Further, binding of free drug to the liposome surface causes aggregation and larger liposomes activate the $\mathrm{C}$ system stronger than small ones [27].

Already the early studies disclosed that multilamellar vesicles with high cholesterol content (71 mole \%) caused a drastic drop in blood pressure while administration of the same dose of low cholesterol-containing (43 mole \%) multilamellar vesicles did not change blood pressure [28]. An explanation for the role of cholesterol can be that cholesterol-loaded liposomes bind more to naturally occurring anticholesterol antibodies. In fact, liposomes containing $71 \%$ cholesterol bound 9-fold higher amount of murine monoclonal anticholesterol antibodyies than liposomes containing $43 \%$ cholesterol. Similar results were observed when normal and cholesterol enriched liposomes were incubated with human sera [28].

It is generally observed that negative charge on the

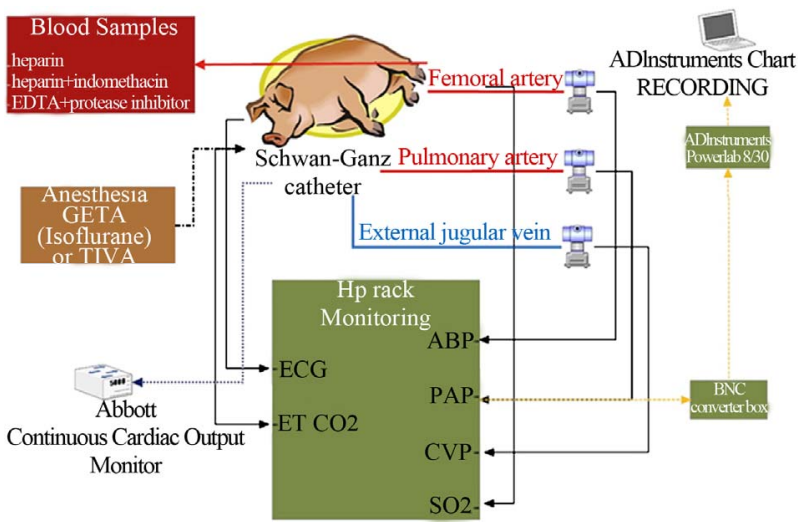

Figure 3. Assessing CARPA and cardiopulmonary distress by nanoparticles in the pig (Szebeni et al. [24]). 
liposome surface increased reactivity. The strongly negative liposomes containing fully hydrogenated soy phosphatidylcholine-cholesterol-soy phosphatidyl-glycerol induced much stronger $C$ activation in human sera than the comparable less negative vesicles [27].

Surface characteristics of liposomes can also alter their $\mathrm{C}$ activating properties. A "structure-activity" relationship of nanoparticles with various surface configurations have shown that alteration from "mushroom" to "brush" surface configuration shifted $\mathrm{C}$ activation from C1q-dependent classical to lectin pathway and also reduced $\mathrm{C}$ activation [29].

Liposomes containing poly(ethylene imine) induced significant and concentration-dependent $\mathrm{C}$ activation whereas its two PEGylated poly(ethylene imine) derivatives were without an effect in human sera. The same difference between the poly(ethylene imine) liposomes and their PEGylated derivatives were demonstrated in the pig model after intravenous administration, showing CARPA, i.e. transient cardiopulmonary distress. It was also found that poly(ethylene imine) liposomes caused both self- and cross-tolerance [30].

\section{SUMMARY AND CONCLUSIONS}

Complement Activation-Related Pseudoallergy (CARPA) is a detrimental reaction of the cardiopulmonary system propelled by the hypersensitive reaction of the immune system in response to various $\mathrm{C}$ activating allergens. It can be clearly differentiated from classical anaphylatoxic reactions, while in contrast to the IgE mediation of classical allergy, activation of the $\mathrm{C}$ system plays a crucial role in the mechanism of action. Pseudoallergic reactions bear important human relevance, due to their high incidence upon food or drug-related exposures, insect bites, or even surgical interventions without prior sensitization. New drug formulations, especially nanomedicinal (e.g. liposomal) products resulted in new challenges because of their CARPA-inducing characteristics.

Cardiovascular and pulmonary symptoms of CARPA are ranging from mild to severe reactions. The latter could result in respiratory and/or cardiac arrest, as well as huge and sudden changes in pulmonary and systemic arterial pressures. The sensitivity to CARPA widely varies among species, the most sensitive are being humans and pigs, while dogs are less sensitive and rodents seem to be relatively insensitive. While this special hypersensitive reaction has important clinical relevance nowadays, animal models of CARPA that are potentially capable of evaluating the pseudoallergic properties of novel drug candidates are of utmost importance. The porcine model of CARPA proved to be the best model so far to predict cardiovascular and pulmonary changes evoked by various drug injections. The only disadvantage of the pig test is that it is rather labor consuming and also expensive; therefore it is inappropriate for large scale screening purposes. Therefore, it still leaves room for the development of other (e.g. rodent) models in order to predict the cardiopulmonary side effects of new chemical entities due to CARPA.

\section{REFERENCES}

[1] Fildes, J.E., Shaw, S.M., Yonan, N. and Williams, S.G. (2009) The immune system and chronic heart failure: Is the heart in control? Journal of the American College of Cardiology, 53, 1013-1020.

doi:10.1016/j.jacc.2008.11.046

[2] Szebeni, J. (2005) Complement activation-related pseudoallergy: A new class of drug-induced acute immune toxicity. Toxicology, 216, 106-121. doi:10.1016/j.tox.2005.07.023

[3] Coombs, R.R.A. and Gell, P.G.H. (1968) Classification of allergic reactions responsible for drug hypersensitivity reactions. In: Coombs, R.R.A. and Gell, P.G.H., Eds., Clinical Aspects of Immunology, Davis, Philadelphia, 575596.

[4] Szebeni, J., Fontana, J.L., Wassef, N.M., Mongan, P.D., Morse, D.S., Dobbins, D.E., Stahl, G.L., Bunger, R. and Alving, C.R. (1999) Hemodynamic changes induced by liposomes and liposome-encapsulated hemoglobin in pigs: A model for pseudoallergic cardiopulmonary reactions to liposomes. Role of complement and inhibition by soluble CR1 and anti-C5a antibody. Circulation, 99, 2302-2309. doi:10.1161/01.CIR.99.17.2302

[5] Szebeni, J., Baranyi, L., Savay, S., Milosevits, J., Bunger, R., Laverman, P., Metselaar, J.M., Storm, G., ChananKhan, A., Liebes, L., Muggia, F.M., Cohen, R., Barenholz, Y. and Alving, C.R. (2002) Role of complement activetion in hypersensitivity reactions to doxil and hynic PEG liposomes: Experimental and clinical studies. Journal of Liposome Research, 12, 165-172.

doi:10.1081/LPR-120004790

[6] Szebeni, J., Bedocs, P., Csukas, D., Rosivall, L., Bunger, R. and Urbanics, R. (2012) A porcine model of complement-mediated infusion reactions to drug carrier nanosystems and other medicines. Advanced Drug Delivery Reviews, 64, 1706-1716. doi:10.1016/j.addr.2012.07.005

[7] Szebeni, J., Bedocs, P., Urbanics, R., Bunger, R., Rosivall, L., Toth, M. and Barenholz, Y. (2012) Prevention of infusion reactions to PEGylated liposomal doxorubicin via tachyphylaxis induction by placebo vesicles: A porcine model. Journal of Controlled Release: Official Journal of the Controlled Release Society, 160, 382-387.

[8] Szebeni, J., Alving, C.R., Rosivall, L., Bunger, R., Baranyi, L., Bedocs, P., Toth. M. and Barenholz, Y. (2007) Animal models of complement-mediated hypersensitivity reactions to liposomes and other lipid-based nanoparticles. Journal of Liposome Research, 17, 107-117. doi:10.1080/08982100701375118

[9] Chenoweth, D.E., Cooper, S.W., Hugli, T.E., Stewart, R.W., Blackstone, E.H. and Kirklin, J.W. (1981) Com- 
plement activation during cardiopulmonary bypass: Evidence for generation of C3a and C5a anaphylatoxins. The New England Journal of Medicine, 304, 497-503. doi:10.1056/NEJM198102263040901

[10] Bruins, P., te Velthuis, H., Yazdanbakhsh, A.P., Jansen, P.G., van Hardevelt, F.W., de Beaumont, E.M., Wildevuur, C.R., Eijsman, L., Trouwborst, A. and Hack, C.E. (1997) Activation of the complement system during and after cardiopulmonary bypass surgery: Postsurgery activation involves C-reactive protein and is associated with postoperative arrhythmia. Circulation, 96, 3542-3548. doi:10.1161/01.CIR.96.10.3542

[11] Garay, R.P., El-Gewely, R., Armstrong, J.K., Garratty, G. and Richette, P. (2012) Antibodies against polyethylene glycol in healthy subjects and in patients treated with PEG-conjugated agents. Expert Opinion on Drug Delivery, 9, 1319-1323. doi:10.1517/17425247.2012.720969

[12] Brouwers, A.H., De Jong, D.J., Dams, E.T., Oyen, W.J., Boerman, O.C., Laverman, P., Naber, T.H., Storm, G. and Corstens, F.H. (2000) Tc-99m-PEG-Liposomes for the evaluation of colitis in Crohn's disease. Journal of Drug Targeting, 8, 225-233. doi:10.3109/10611860008997901

[13] Chanan-Khan, A., Szebeni, J., Savay, S., Liebes, L., Rafique, N.M., Alving, C.R. and Muggia, F.M. (2003) Complement activation following first exposure to pegylated liposomal doxorubicin (Doxil): Possible role in hypersensitivity reactions. Annals of Oncology, 14, 1430-1437. doi:10.1093/annonc/mdg374

[14] Ulevitch, R.J., Cochrane, C.G., Henson, P.M., Morrison, D.C. and Doe, W.F. (1975) Mediation systems in bacterial lipopolysaccharide-induced hypotension and disseminated intravascular coagulation. I. The role of complement. The Journal of Experimental Medicine, 142, 1570-1590. doi:10.1084/jem.142.6.1570

[15] Ulevitch, R.J. and Cochrane, C.G. (1978) Role of complement in lethal bacterial lipopolysaccharide-induced hypotensive and coagulative changes. Infection and Immunity, 19, 204-211.

[16] Ulevitch, R.J. and Cochrane, C.G. (1977) Complementdependent hemodynamic and hematologic changes in the rabbit. Inflammation, 2, 199-216. doi:10.1007/BF00917596

[17] Lundberg, C., Marceau, F. and Hugli, T.E. (1987) C5ainduced hemodynamic and hematologic changes in the rabbit. Role of cyclooxygenase products and polymorphonuclear leukocytes. The American Journal of Pathology, 128, 471-483.

[18] Damas, J. and Lagneaux, D. (1991) Dissociation between the effects of zymosan on the systemic and pulmonary vessels of the rat. British Journal of Pharmacology, 104, 559-564. doi:10.1111/j.1476-5381.1991.tb12468.x

[19] Rabinovici, R., Rudolph, A.S. and Feuerstein, G. (1989) Characterization of hemodynamic, hematologic, and biochemical responses to administration of liposome-encapsulated hemoglobin in the conscious, freely moving rat. Circulatory Shock, 29, 115-132.

[20] Rabinovici, R., Rudolph, A.S. and Feuerstein, G. (1990) Improved biological properties of synthetic distearoyl phosphatidyl choline-based liposome in the conscious rat. Circulatory Shock, 30, 207-219.

[21] Rabinovici, R., Rudolph, A.S., Yue, T.L. and Feuerstein, G. (1990) Biological responses to liposome-encapsulated hemoglobin (LEH) are improved by a PAF antagonist. Circulatory Shock, 31, 431-445.

[22] Szebeni, J., Wassef, N.M., Spielberg, H., Rudolph, A.S. and Alving, C.R. (1994) Complement activation in rats by liposomes and liposome-encapsulated hemoglobin: Evidence for anti-lipid antibodies and alternative pathway activation. Biochemical and Biophysical Research Communications, 205, 255-263. doi:10.1006/bbrc.1994.2658

[23] Szebeni, J., Spielberg, H., Cliff, R.O., Wassef, N.M., Rudolph, A.S. and Alving, C.R. (1997) Complement activation and thromboxane secretion by liposome-encapsulated hemoglobin in rats in vivo: Inhibition by soluble complement receptor type 1. Artificial Cells, Blood Substitutes, and Immobilization Biotechnology, 25, 347-355. doi:10.3109/10731199709118925

[24] Szebeni, J., Muggia, F., Gabizon, A. and Barenholz, Y. (2011) Activation of complement by therapeutic liposomes and other lipid excipient-based therapeutic products: Prediction and prevention. Advanced Drug Delivery Reviews, 63, 1020-1030. doi:10.1016/j.addr.2011.06.017

[25] Wassef, N.M., Johnson, S.H., Graeber, G.M., Swartz, G.M., Jr., Schultz, C.L., Hailey, J.R., Johnson, A.J., Taylor, D.G., Ridgway, R.L. and Alving, C.R. (1989) Anaphylactoid reactions mediated by autoantibodies to cholesterol in miniature pigs. Journal of Immunology, 143, 2990 2995.

[26] Szebeni, J., Baranyi, L., Savay, S., Bodo, M., Milosevits, J., Alving, C.R. and Bunger, R. (2006) Complement activation-related cardiac anaphylaxis in pigs: Role of C5a anaphylatoxin and adenosine in liposome-induced abnormalities in ECG and heart function. American Journal of Physiology. Heart and Circulatory Physiology, 290, H1050-H1058. doi:10.1152/ajpheart.00622.2005

[27] Szebeni, J., Bedocs, P., Rozsnyay, Z., Weiszhar, Z., Urbanics, R., Rosivall, L., Cohen, R., Garbuzenko, O., Bathori, G., Toth, M., Bunger, R. and Barenholz, Y. (2012) Liposome-induced complement activation and related cardiopulmonary distress in pigs: Factors promoting reactogenicity of Doxil and AmBisome. Nanomedicine: Nanotechnology, Biology, and Medicine, 8, 176-184. doi:10.1016/j.nano.2011.06.003

[28] Baranyi, L., Szebeni, J., Savay, S., Bodo, M., Basta, M., Bentley, T.B., Bunger, R. and Alving, C.R. (2003) Complement-dependent shock and tissue damage induced by intravenous injection of cholesterol-enriched liposomes in rats. The Journal of Applied Research in Clinical and Experimental Therapeutics, 3, 1-19.

[29] Hamad, I., Al-Hanbali, O., Hunter, A.C., Rutt, K.J., Andresen, T.L. and Moghimi, S.M. (2010) Distinct polymer architecture mediates switching of complement activation pathways at the nanosphere-serum interface: Implications for stealth nanoparticle engineering. ACS Nano, 4, 66296638. doi:10.1021/nn101990a 
[30] Merkel, O.M., Urbanics, R., Bedocs, P., Rozsnyay, Z., Rosivall, L., Toth, M., Kissel, T. and Szebeni, J. (2011) In vitro and in vivo complement activation and related anaphylactic effects associated with polyethylenimine and polyethylenimine-graft-poly(ethylene glycol) block copolymers. Biomaterials, 32, 4936-4942.

doi:10.1016/j.biomaterials.2011.03.035 